\title{
LA COMMUNICATION DES MUSÉES D'ENTREPRISE EN PROVENCE ET LA CONSTRUCTION DE LA PROVENÇALITÉ
}

\author{
Lise Renaud $^{1}$ et Laure Marchis-Mouren ${ }^{2}$
}

Face à un besoin de transformation du discours publicitaire, le musée d'entreprise pourrait bien être une nouvelle stratégie communicationnelle permettant d'allier arguments culturels et commerciaux. En intégrant une exposition patrimoniale à un espace de promotion des produits commerciaux, ces institutions deviennent des acteurs de la patrimonialisation. Mais comment arrivent-elles à articuler culture et économie ? Comment les stratégies patrimoniales et institutionnelles s'articulent-elles ? S'entremêlent-elles ou cohabitent-elles ?

Cet article développe l'idée que le fonctionnement sémiotique de la communication des musées d'entreprise permet de porter un double discours sans contradiction apparente. À partir de l'étude de quatre musées d'entreprise situés en Provence, quatre stratégies énonciatives de valorisation d'une organisation sont identifiées : s'inscrire dans un temps long, refléter un territoire historique et culturel, offrir des trésors à la contemplation, être le garant d'une éthique patrimoniale.

1 Lise Renaud est maître de conférences au département information communication, université d'Avignon et des Pays de Vaucluse, Équipe Culture et Communication du Centre Norbert Elias (UMR 8562).

2 Laure Marchis-Mouren est docteure au département information communication, université d'Avignon et des Pays de Vaucluse, Équipe Culture et Communication du Centre Norbert Elias (UMR 8562).

Recherches en communication, $\mathrm{n}^{\circ} 45$ - Article publié le 23/05/2018 
Depuis les années 90, le statut de musée d'entreprise interroge quant à sa légitimité culturelle et patrimoniale (De La Broise, 1996 ; Rasse, 1995). S'il est évident que son émergence relève davantage d'enjeux économiques que culturels, les formes de cette institution demeurent hybrides et équivoques (Cousserand, 2009). Ainsi à travers les mises en scène patrimoniales et la mise en exposition d'une collection singulière, les entreprises construisent et diffusent une image dont l'opérativité symbolique repose sur les codes des musées et de leurs collections patrimoniales, ce qui semble leur permettre de s'éloigner du registre publicitaire et pire commercial.

Or, si la collection exposée peut revêtir un certain prestige patrimonial, elle efface les frontières. La proximité, voire l'intégration d'une exposition patrimoniale à un espace de promotion des produits commerciaux de l'entreprise la plonge dans l'équivoque. Les locaux d'exposition à vocation culturelle proches ou associés à ceux dédiés au commerce et à la vente construisent une image singulière de l'organisation. La question qui se pose alors est d'identifier comment les discours informationnels ou culturels s'hybrident avec les arguments publicitaires ou de promotion des ventes. Les logiques de valorisation patrimoniales et institutionnelles s'entremêlent-elles ou cohabitent-elles ? Sontelles antagonistes ou synergiques?

Afin d'éclairer ce questionnement, cet article se base sur une analyse sémiotique de supports de communication externe et de visites expertes de quatre musées d'entreprise situés en Provence. Ce travail d'identification des représentations et des modes de figuration à la fois des objets patrimoniaux, des savoirs et des publics-visiteurs nous a permis de repérer des arguments de valorisation récurrents quelles que soient les caractéristiques de l'organisation étudiée.

Ce travail d'investigation nous suggère de distinguer quatre références des modalités de valorisation à même d'expliciter le prestige et l'attraction des marchandises mises sur le marché par les firmes qui ont choisi d'investir dans la création d'un musée : la valeur d'ancienneté par la figuration du passé, la valeur territoriale par référence à une localité, la valeur de trouvaille à travers les 
figures du trésor et la valeur de préservation par évocation d'une mission désintéressée de transmission de la provençalité.

En définitive, c'est cette construction identitaire que progressivement le musée d'entreprise a su imposer. Il apparaît comme un lieu-ressource de valorisation d'une identité souhaitée dans un contexte dans lequel on assiste à une transformation du discours publicitaire.

\section{Le musée d'entreprise comme opérateur symbolique de l'image externe d'une organisation marchande}

S'il réside un flottement pour recenser et circonscrire les musées d'entreprise notre attention. Si l'instauration d'un musée d'entreprise permet d'affirmer la mission sociale et culturelle de l'organisation tout en créant une image de marque, alors identifier par quels procédés sémiotiques ces objectifs cherchent à être atteints devient essentiel pour comprendre l'engouement des entreprises envers le développement de cette forme organisationnelle. Comment ces stratégies communicationnelles fonctionnent-elles ? Sur quoi s'appuient-elles pour opérer?

Ainsi, d'une part, nous faisons le choix dans cette recherche de nous restreindre à la définition du musée d'entreprise proposée par Desvallées et Mairesse, à savoir une " catégorie de musées de nature essentiellement privée et liée à une entreprise encore en activité, destinée à la préservation et la mise en valeur de l'histoire de cette entreprise depuis sa fondation, notamment à partir des produits qu'elle a réalisés » (Desvallées \& Mairesse, 2011, p. 628) et de nous focaliser dans cet ensemble sur ceux instaurés par une organisation commerciale et ouverts au grand public. En somme, nous nous intéressons au modèle canonique du musée d'entreprise.

D'autre part, nous nous concentrons sur la relation entre les mises en scène déployées à travers le discours porté par le musée d'entreprise et la stratégie d'image, d'identification externe de l'organisation. L'analyse du discours d'un musée d'entreprise repose dans ce cas sur une conception de la patrimonialisation d'une marque ou d'une industrie et de la mise en exposition 
d'une collection associée à une entreprise comme un processus profondément communicationnel et symbolique (Davallon, 2006). L'exposition peut en effet se définir comme la forme médiatique la plus aboutie de la patrimonialisation (De La Broise, 1996). Ainsi cette étude s'inscrit dans une réflexion sur les enjeux pour une organisation de développer une stratégie de communication (la patrimonialisation) qui lui permet de transférer et de s'associer des valeurs patrimoniales à même d'effacer ou d'édulcorer son rapport économique.

\section{2. Étudier la communication des musées d'entreprise en Provence : une entrée par la figuration et la valorisation}

Suivant cette perspective, nous avons réalisé une étude sémiotique de la communication externe (supports de communication et visite-experte) de quatre musées d'entreprise situés en Provence : le musée du savon de Marseille (Marius Fabre), le musée de la lavande (Lincelé), le musée du textile provençal (Souleiado), le musée l'Occitane.

Le choix de ce terrain a été induit par un contexte pédagogique. Nous avons toutes les deux, respectivement en 2015 et 2016, élaboré et dispensé un cours durant lequel a été déployé un travail avec des étudiants de première année de master muséologie ${ }^{3}$ visant à leur permettre de confronter la promesse de visite d'un musée d'entreprise (au total huit musées ont été étudiés), celle évoquée à travers les supports de communication externe, à une visite sur place tout en leur permettant de saisir l'ambiguïté identitaire de ces musées de marque. Or au cours de cet exercice et bien que chaque groupe d'étudiants ait travaillé sur un seul musée, nous avons pu constater des récurrences dans les arguments de valorisation patrimoniale.

3 Nous remercions les étudiants du Master Médiations de la Culture et des Patrimoines de l'université d'Avignon pour leur investissement dans le cadre de cet enseignement et pour nous avoir permis, à travers les discussions et rendus, de prendre la mesure des stratégies de valorisation et de figuration développés par les musées d'entreprise de Provence. 
Ce constat nous a amené à procéder à une nouvelle étude, cette fois comparée, et dont l'objectif était de repérer les procédés énonciatifs de figuration utilisés, mais aussi les valeurs véhiculées dans le discours de l'institution tant au niveau des supports de communication externe que de l'exposition. Une série de questions nous a guidées dans ce travail : dans ces musées, qu'estce qui est patrimonialisé ? Quelle muséographie donne à voir ce patrimoine? Comment, dans le propos d'exposition, sont intégrés l'organisation et ses produits ? Quelle image de l'entreprise est ainsi construite et quelles valeurs lui sont associées ?

Pour répondre à ces interrogations, nous avons élaboré une grille d'analyse sémiotique pour repérer les connotateurs, c'està-dire les signifiants de connotation (Barthes, 1964), et valeurs mobilisées dans les documents de communication et avons procédé à des relevés photographiques lors des visites expertes soumis ensuite à analyse afin d'étudier la nature des expôts ${ }^{4}$ c'est-à-dire des unités élémentaires d'exposition, le contenu et forme des outils de médiation, l'agencement de la collection, le parcours de visite, autant d'éléments qui contribuent à construire et exprimer le discours de l'exposition d'un musée d'entreprise.

Précisons que l'agencement de la grille permet pour chaque valeur repérée (en ligne dans le tableau) d'indiquer les signes utilisés pour l'évoquer ; par exemple le naturel est connoté par les couleurs et la nature du support. Les colonnes sont quant à elles réservées à chaque musée.

Afin d'adopter pour l'étude des documents de communication un regard systématique, le remplissage de la grille a été effectué en respectant le principe d'une décomposition sémiotique, c'est-à-dire en observant chaque type de signe composant le message construit dans les documents de communication : plastique (couleur, forme, support, cadrage, angle de prise de vue, composition), iconique (motif, style),

4 Le terme d'« expôt » est un néologisme proposé par André Desvallées en 1976 en tant que traduction littérale du terme anglais exhibit employé par Duncan Cameron dans sa classification des composants de l'exposition. $C f$. André Desvallées, François Mairesse (dir.), Dictionnaire encyclopédique de muséologie, Armand Colin, 2011, p. 601. 
linguistique. Pour l'étude du discours de l'exposition, ce sont les indicateurs précédemment cités sur lesquels nous nous sommes systématiquement focalisées.

\section{Ressources symboliques de la patrimonialisation}

À l'issue de cette étude, la logique discursive adoptée par les quatre musées étudiés indique la récurrence de stratégies de valorisation à travers la muséalisation et la patrimonialisation de l'activité des entreprises concernées.

Tout d'abord, chaque lieu a recours à un ensemble de codes formels présents dans les expositions muséales permettant d'un point de vue muséographique de les rapprocher des écomusées, des musées de sciences ou de société. Ces quatre entreprises ont en effet investi dans l'aménagement d'un espace d'exposition plus ou moins proche de leur lieu de production ou de fabrication dans lequel se déploie un agencement d'objets assimilés, à travers la scénographie, à une collection, accompagnés de cartels et panneaux, et où le public est invité à suivre un parcours durant lequel divers outils de médiation (brochures, films, ou visites guidées, etc.) sont proposés. Considérant les dispositifs de mise en communication et de mise en exposition de ces lieux, les outils et techniques employés peuvent être rapprochés et assimilés à ceux usités dans les musées classiques. C'est en jouant sur et en empruntant aux codes formels du langage de l'exposition que les musées d'entreprise se donnent à voir comme un musée.

Toutefois deux distinctions notables sont à effectuer. D'une part, la place importante accordée à l'espace de vente par rapport à celui de l'exposition indique l'importance de l'activité économique pour l'organisation et tend à montrer le sens du mouvement dans lequel se déploie le musée d'entreprise de l'économie vers la culture. Par exemple, la boutique du musée de la lavande accessible à l'issue de la visite du musée et indépendamment de celui-ci, a une taille identique à l'espace dédié à l'exposition. De même, le dépliant de présentation du musée Souleiado comporte un volet dédié à la présentation des $300 \mathrm{~m}^{2}$ de magasin d'usine qui font face au musée. En d'autres 
termes, si les musées et sites patrimoniaux sont de plus en plus incités à accroître leurs ressources propres (Tobelem, 2005) et de fait développent leurs espaces de boutique souvenirs, les musées d'entreprise à l'inverse adjoignent à des lieux de vente ou de fabrication des espaces culturels de même envergure.

D'autre part, dans l'ensemble des musées étudiés, la nature des objets exposés (datation, provenance, statut) n'est pas systématiquement précisée. Les informations scientifiques sur les expôts restent très parcellaires. Cette faiblesse des indications tend à donner l'impression qu'associer, voire accumuler, puis exposer des objets anciens dans des vitrines serait suffisant pour faire de cet ensemble d'objets une collection.

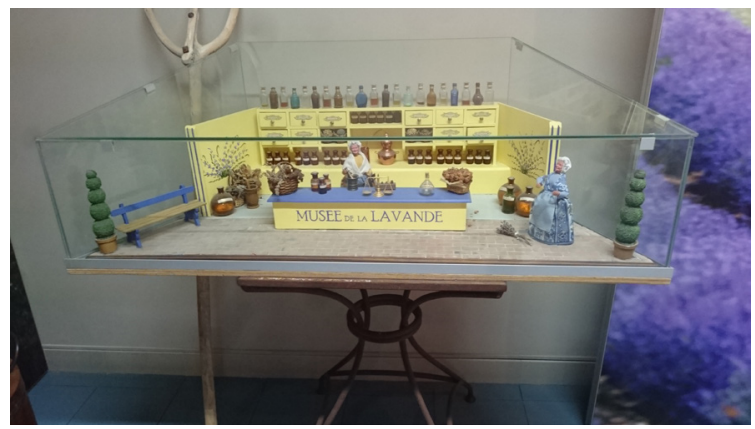

1a. Maquette sans étiquette - Musée Lincelé

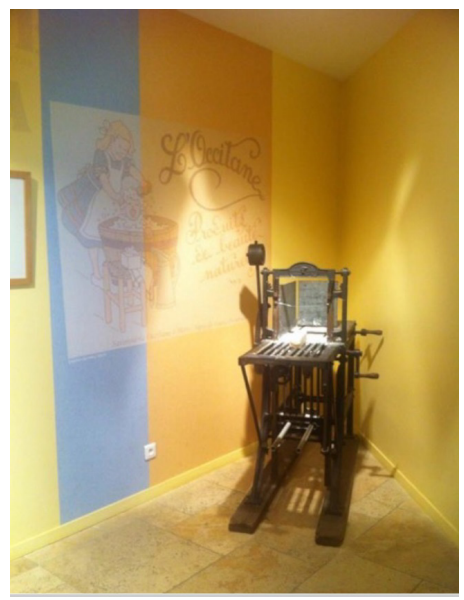

1b. Machine sans étiquette - Musée l'Occitane

Figure 1. Une médiation parcellaire 
Ainsi, le musée d'entreprise, certes, épouse la forme du musée et les codes de son langage d'exposition de ses collections, mais se distingue par la place réservée aux savoirs scientifiques sur les objets exposés et donc par la construction du sens de l'exposition. Le liant au sein de la collection - ce qui fait sens pour le visiteurne repose pas sur la valeur scientifique et historique des objets, mais sur leur relation avec les caractéristiques identitaires de l'entreprise qu'elles relèvent de son histoire, de ses techniques de production ou de la composition de ses produits.

\subsection{La valeur du passé}

Que ce soit dans leur support de communication ou dans le discours qu'ils tiennent dans l'espace muséal, les musées d'entreprise mettent en exergue la dimension temporelle et historique des objets de leur collection, de l'ouverture au public de leur espace d'exposition voire de l'organisation commerciale auquel ils sont rattachés. Deux stratégies énonciatives qui contribuent à produire cette omniprésence du temps passé méritent d'être ici présentées.

Au niveau lexical tout d'abord, les mentions de dates, de périodes historiques, de temps écoulé, sont récurrentes dans les supports de communication. Sur les plaquettes de présentation des musées, le public potentiel peut ainsi lire : " fondée en 1988 », « créée il y a plus de 200 ans », « traversé le $19^{\mathrm{e}}$ siècle et les grandes révolutions industrielles », « la fin du $19^{\mathrm{e}}$ siècle » (musée Souleiado) ; «Depuis 1991 », « un alambic itinérant de 1900 » (musée Lincelé), « Créée en 1976 » (musée l’Occitane); " Depuis 1900 et quatre générations familiales », " 19002010 », « 110 ans » (musée Marius Fabre). Ces mentions écrites constituent des indicateurs temporels qui opèrent comme autant de garants du caractère patrimonial du lieu et de son contenu. Exprimer un lien avec le passé est une manière de s'inscrire dans l'histoire. Toutefois, insistons ici sur le mélange ou la confusion des référents auxquels sont attachées ces précisions temporelles. Les datations peuvent renvoyer aux objets de la collection comme pour certifier la valeur historique de cette dernière (par 
exemple on peut relever : " importante collection de textiles et de costumes traditionnels des $18^{\mathrm{e}}, 19^{\mathrm{e}}$ et $20^{\mathrm{e}}$ siècles $»$ (plaquette du musée Souleiado) ou « la plus importante collection d'alambics à lavande en cuivre rouge du $16^{\mathrm{e}}$ siècle à nos jours » (plaquette du musée Lincelé)). Mais les inscriptions de dates sont surtout utilisées pour se référer à la création du musée, de l'entreprise ou de la marque. En d'autres termes, à travers cet usage des mentions temporelles, un flou est instauré sur ce qui fait patrimoine. Ce mélange permet finalement des jeux de transferts symboliques entre les objets exposés, le musée, l'entreprise et la marque. Par ce biais, les valeurs d'authenticité et de durabilité sont adjointes à l'organisation marchande et ses produits.

L'entreprise, la marque, donne à voir sa capacité à durer dans le temps, sa longévité, tout en confirmant son inscription dans l'ère contemporaine. Les bâtiments sont récents, la muséographie épurée, les dispositifs de médiations empreints de modernité. Le musée de l'Occitane dispose d'un grand écran interactif sur lequel le visiteur peut sélectionner les histoires à écouter pour chaque plante utilisée pour ses huiles essentielles. Au musée de la lavande, un diffuseur de parfum permet au visiteur d'identifier successivement l'odeur de la lavande puis du lavandin. Seul le musée Souleiado, installé dans la "résidence de la manufacture Souleidado depuis deux siècles » d'après le site internet, met en scène l'ancienneté du bâti pour servir le message d'authenticité délivré par l'entreprise.

Ainsi, la stratégie énonciative adoptée vise à démontrer que ces musées ne présentent pas un produit ou une technique de production, mais bien des objets patrimoniaux matériels et immatériels qui méritent à ce titre d'être conservés et transmis aux générations futures. Ces derniers n'ont plus seulement une valeur utilitaire et économique, ils ont surtout une valeur patrimoniale (transformation symbolique). Le passé est alors à voir en ce sens comme un gisement de valeurs pour l'organisation commerciale et ses produits. 


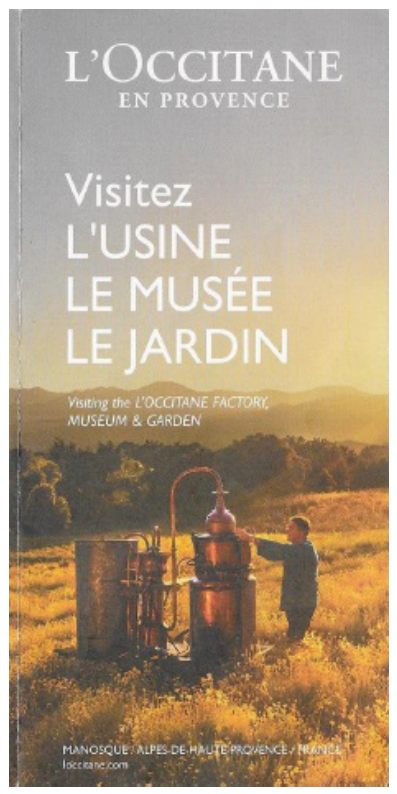

2a. Premier volet du dépliant du musée l'Occitane

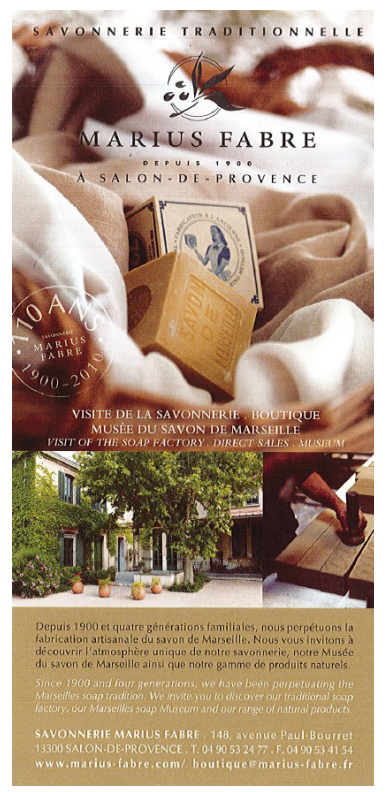

2b. Recto du dépliant du musée Marius Fabre

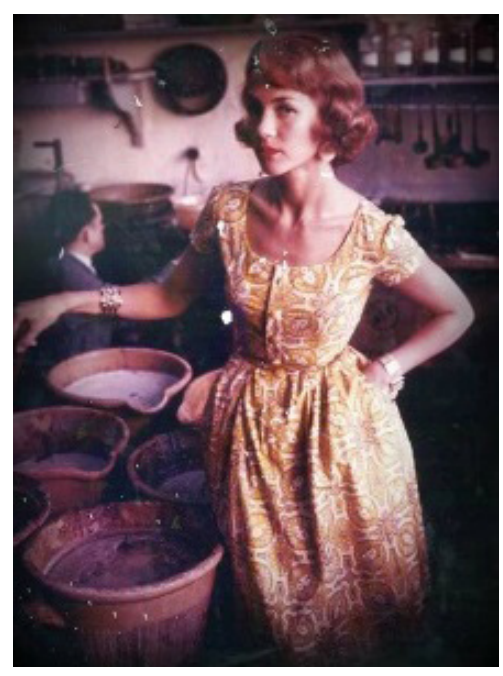

2c. Illustration photographique issue du site web du musée Souleiado

Figure 2. Évocation du passé par les mises en forme des supports de communication 
La deuxième stratégie énonciative d'association au temps passé repose sur la forme de la communication. Le design graphique des supports de communication - voire aussi la scénographie des lieux d'exposition, par exemple au musée du savon de Marseille où l'exposition se déploie dans une vieille salle de séchage - donne à percevoir un aspect ancien. Souleiado a fait le choix d'illustrer ses pages web de présentation du musée avec des photographies dont l'intensité des couleurs, la texture ou le format expriment des temps reculés. Si la plaquette du musée l'Occitane comporte une première page qui évoque les peintures réalistes de la fin du $19^{\mathrm{e}}$ siècle (photographie d'un homme à côté d'un alambic en cuivre dans un champ d'immortelles au lever du soleil), celle du musée Marius Fabre suggère l'ancien et le naturel à travers ses couleurs (tons marron-beige-vert kaki) et sa texture (papier demi-mat, granuleux avec un grammage de $170 \mathrm{~g} / \mathrm{m} 2$ ).

Ainsi, la communication des musées étudiés joue au niveau formel avec la valeur d'ancienneté au sens de Riegl (Riegl, 1903) comprise comme la monstration du passage du temps, qui opère comme un garant de l'authenticité du lieu, et par métonymie de sa collection, voire plus indirectement de son discours. Le passé semble ici exploité pour sa capacité à renvoyer à la tradition et l'artisanat. Quand sont évoqués "un savoir-faire ancestral » (Marius Fabre) ou « la Provence d'antan » (Souleiado), l'imagerie suggérée n'est pas celle de la production industrielle, mais davantage le fantasme d'une fabrication naturelle et artisanale, celui d'un passé sublimé. À ce niveau, réside un paradoxe qui constitue un véritable enjeu pour le discours des organisations marchandes : s'associer au passé, à une image artisanale en ouvrant un musée oblige aussi l'entreprise à s'éloigner d'un rapprochement avec l'innovation et le progrès. L'Occitane, tout comme Souleiado, essaye de concilier les deux versants, mais au risque de produire parfois des discours contradictoires.

\subsection{La valeur territoriale}

Le thème de l'ancrage territorial est un autre argument de valorisation de l'activité muséale récurrent. Le public le retrouve 
convoqué tant dans les supports de communication que dans les espaces d'exposition. Les nombreuses mentions géographiques dans les dépliants et sur les sites internet que nous avons pu relever peuvent avoir comme objectif pratique de préciser la localisation du musée en indiquant une ville (Aix-en-Provence, Manosque, Tarascon, Coustellet). Ils sont d'ailleurs généralement accompagnés d'un plan.

Toutefois, l'utilisation fréquente du nom « Provence » ou de l'adjectif « provençal », ainsi que sa mise en exergue dans la titraille, revêt à notre avis une tout autre logique stratégique. Les quatre musées étudiés sont certes bien localisés dans la région historique "Provence ». Leurs communes d'implantation ont chacune fait partie du territoire provençal à un moment donné de leur histoire. Mais c'est moins la dimension géographique que la dimension symbolique que ces institutions cherchent à convoquer en utilisant cette terminologie. Le nom «Provence» est un réservoir d'imaginaires et d'imageries dont les organisations marchandes peuvent tirer bénéfice pour sublimer leur production et produits. Son caractère patrimonial est par ailleurs collectivement et touristiquement reconnu, ce qui permet de surcroît de renforcer la crédibilité et la légitimité de la valeur des collections des musées d'entreprise.

Ce travail d'évocation du patrimoine de Provence peut se voir renforcé par le design graphique des plaquettes et la scénographie des lieux (l'usage du violet par le musée de la lavande est à ce niveau symptomatique de ce travail d'évocation d'images), mais aussi par des stratégies plus indirectes comme l'utilisation du dialecte provençal sur la première page de la plaquette du musée Souleiado. 


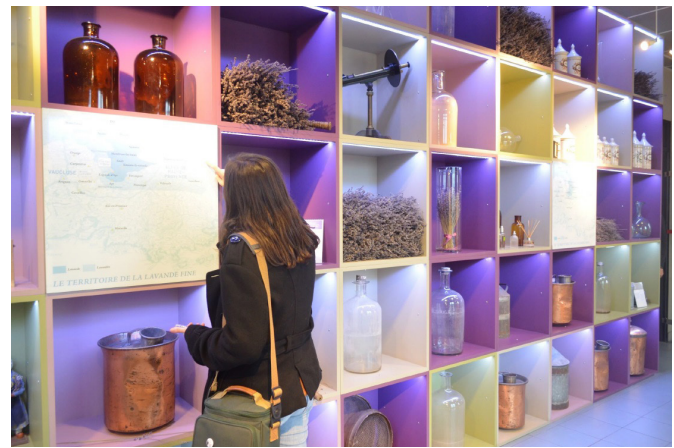

3a. Scénographie entrée du musée de la lavande

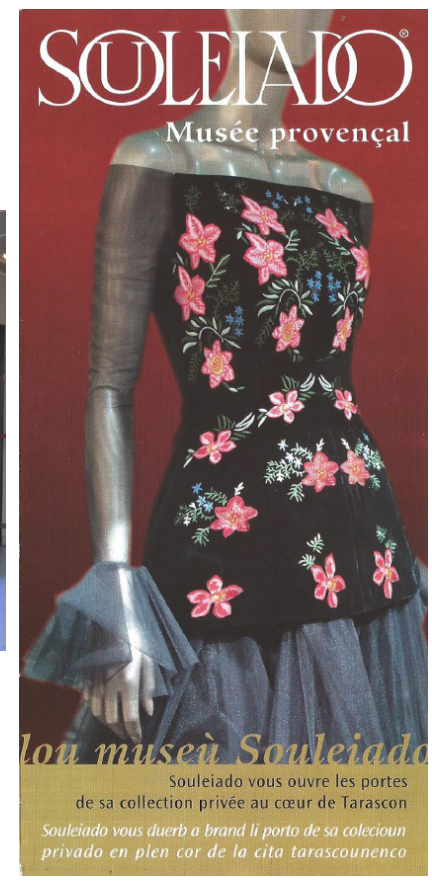

3b. Premier volet du musée Souleiado

Figure 3. Stratégies d'évocation du patrimoine de Provence

On peut ainsi s'avancer à dire que non seulement le musée est localisé en Provence, mais surtout l'activité de l'entreprise dont il relève, utilise des produits de cette région qui sont pour certains des symboles de ce territoire comme la lavande ou l'olivier (dont une branche stylisée compose le logo de Marius Fabre). Ainsi, le discours du musée consiste à souligner cet ancrage et attachement territorial. Les références à la culture locale au sein de l'exposition renforcent ce discours à l'instar du Musée Souleiado qui a proposé en 2016 et 2017 une exposition temporaire sur le thème « 50 ans de feria ». Le clin d'œil à la culture taurine, ancrée sur le territoire, devient évident lorsque l'on connait l'importance des 
manifestations taurines lors de ces événements ${ }^{5}$. Et ce d'autant plus qu'une partie des vêtements vendus par Souleido s'inspirent des coupes traditionnelles des gardians, les assistants des éleveurs de taureaux (modèle « veste gardian »).

L'histoire de l'entreprise racontée par le musée est rattachée aux spécificités agricoles et aux savoir-faire du terroir provençal. L'entreprise se positionne de fait comme reflétant par son activité un patrimoine naturel, régional et territorial. Par exemple, Marius Fabre indique sur son site internet que « La Provence devient dès le Moyen Âge la région du savon par excellence ». Quant au musée de la lavande, son film introductif indique que la lavande est une « plante indissociable de la Provence » et sa plaquette que « la véritable lavande de Provence a trouvé son écrin ». Dès lors, les musées étudiés s'appuient sur une vision de la Provence en tant que patrimoine naturel et agricole, et en même temps participent à sa patrimonialisation. On assiste à un double mouvement de reconnaissance. Il n'est d'ailleurs pas anodin qu'un des onglets du musée Marius Fabre soit consacré à la découverte de la région. Celui-ci délivre des informations et conseils touristiques et débute par le titre « Plongez au cœur de la Provence $»^{6}$.

\subsection{La valeur de trouvaille}

Une autre logique de valorisation commune à l'ensemble des musées étudiés est celle de la mise en évidence du caractère d'exception de ce qui est présenté au public dans le musée et de l'expérience qu'il pourra en retirer. Si cette stratégie n'est pas spécifique au type de musée étudié - il est en effet fréquent qu'un site patrimonial effectue sa promotion en promettant une expérience de visite inoubliable - nous considérons que cette

5 Les férias sont des événements populaires organisés par les municipalités dans le sud-est et le sud-ouest de la France. Pendant plusieurs jours, la population locale et touristique se réunit dans divers lieux publics et privés : dans la rue, dans les bodegas (bars privés). Les animations et spectacles rythment la féria. Les événements taurins sont privilégiés : corridas dans les arènes, courses de taureaux. Il y a aussi des chants et des danses traditionnelles. Les rues sont animées par des peňas (fanfares). Les bars tiennent des comptoirs dans la rue (le vin et la bière sont les boissons les plus consommées).

6 http://www.musee-savon-marseille.com/decouvrir-la-region/ 
logique énonciative vise pour les musées d'entreprise à attester du caractère patrimonial de leur collection (et du musée).

Ce besoin de souligner la rareté de ce qui est exposé, d'en faire un trésor auquel le musée permet d'accéder (" atmosphère unique » (Marius Fabre), " musée atypique " « sa collection privée ", " ce musée fait découvrir de multiples trésors » (Souleiado), " L'exceptionnel », « mystère d'alchimiste ", « la magie est présente " (Lincelé)) est un moyen de donner à la collection le statut de trouvaille et donc de la faire entrer dans le processus de patrimonialisation (Davallon, Ibid.).

Relevons en outre que cette sacralisation de la trouvaille, cette mise en exergue du trésor, semble dans le cas des musées d'entreprise être particulièrement utile, car elle est à même de pouvoir se déplacer sur le savoir-faire et donc les secrets de fabrication. In situ, la préciosité des expôts est matérialisée par les lumières et les vitrines qui protègent parfois des objets pourtant très récents et produits en millions d'exemplaires, à l'image des produits et des packagings l'Occitane.

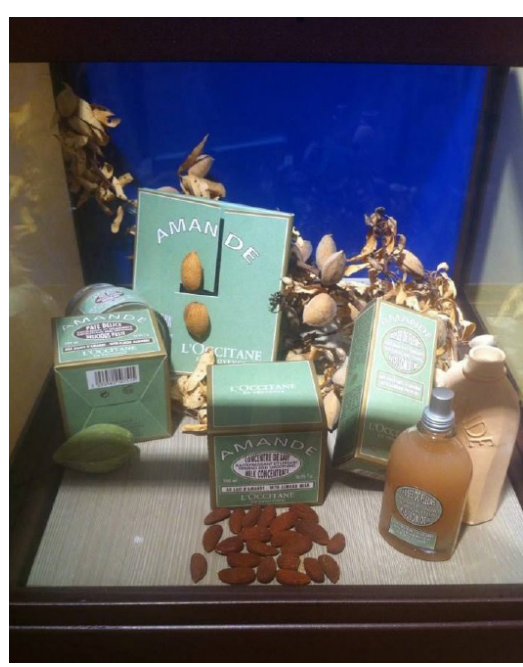

4a. Scénographie vitrine « Amande » 1'Occitane Figure

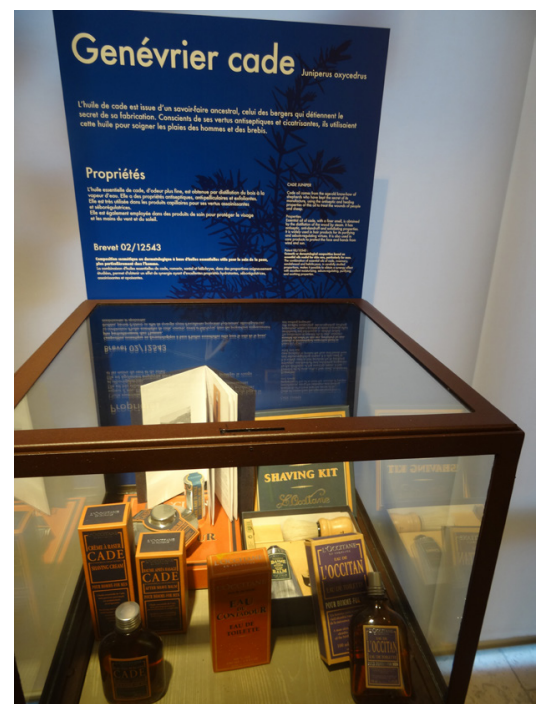

Vitrine 1'Occitane avec cartel

Figure 4. Mise en vitrine des produits-expôts l'Occitane 


\subsection{La valeur de préservation de la provençalité}

La dernière stratégie énonciative commune est celle de l'inscription du discours des musées d'entreprise dans une politique de responsabilité sociale de l'organisation. D'après notre analyse, la communication muséale travaille à démontrer combien l'entreprise, son histoire et ses techniques de fabrication œuvrent dans le respect de l'environnement et la préservation du terroir provençal. Les mentions à ces politiques entrepreneuriales sont récurrentes dans les supports de communication et tout au long des expositions (voire même en boutique). Le film d'introduction au musée de la lavande insiste par exemple sur le rôle de la famille Lincelé dans la préservation de la lavande menacée par les industries chimiques. Le musée est ainsi désigné comme «l'exceptionnel conservatoire de la lavande fine de Provence ». Il est le résultat de l'engagement de cette organisation marchande. Le musée l'Occitane positionne aussi sa marque dans une logique écologique et de production responsable (production locale ou éthique pour le beurre de karité) tout en masquant des caractéristiques plus discutables comme l'empreinte carbone de ses exportations (l'Occitane réunit en effet près de 3000 boutiques dans 90 pays dont Japon, États-Unis, Hong Kong, Chine qui représentent les principales recettes de son chiffre d'affaires en 2016 (plus de 50\%) contre moins de $8 \%$ pour la France).

Dans le musée, l'exposition d'une collection devient en ce sens le support de la mise en scène de la mission écologique, sociale et culturelle de l'entreprise. L'engagement de l'organisation peut alors être présenté de façon explicite et intégré au discours de l'exposition (comme le panneau dédié à l'aide au Sahel dans l'espace d'exposition du musée l'Occitane qui présente le partenariat de la marque avec la fondation Daniel Balavoine). Il peut aussi être plus diffus à travers l'évocation d'une politique de sauvegarde d'espèces naturelles (la lavande) ou de techniques de fabrication (fabrication de savon de Marseille en chaudron). Ainsi tout en passant au second plan sa mission économique et commerciale, l'organisation se voit valorisée dans sa politique environnementale et culturelle, car elle se présente comme 
le dépositaire d'un patrimoine provençal qu'elle contribue à sauvegarder et valoriser.

Si le discours muséal et de responsabilité sociale de l'organisation est en adéquation, cette osmose ne doit pas donner à croire que l'entreprise agit de façon désintéressée même si elle le présente comme tel. Comme on peut le constater pour la communication responsable ou verte, les actions environnementales ou éthiques d'une entreprise sont une ressource argumentative à même d'alimenter les stratégies communicationnelles de l'organisation et donc de la valoriser de façon indirecte (Renaud, 2014). La communication muséale (la patrimonialisation) répond à notre avis parfaitement aux enjeux contemporains de modification de formes de la communication promotionnelle. En d'autres termes, pour une entreprise, faire valoir son engagement environnemental, social et grâce à un musée, son investissement culturel et patrimonial, produit un réservoir d'images et de valeurs mobilisées et mobilisables dans le cadre de la stratégie de communication globale de l'organisation.

\section{Rénover la communication d'entreprise grâce au musée}

Construire un musée ou exposer une collection patrimoniale pour une entreprise est une démarche qui impacte inévitablement l'image et les valeurs associées à cette organisation. Dans le corpus analysé, quatre stratégies énonciatives ont pu être identifiées : s'adjoindre de la longévité, refléter un territoire historique et culturel, offrir des trésors à la contemplation, être le garant d'une éthique patrimoniale. Si ces stratégies fonctionnent pour les musées provençaux, elles nous semblent en mesure d'être aussi utilisables par l'ensemble des musées d'entreprise. En effet, ces quatre registres (passé, territoire, rareté et éthique) sont inhérents à toute organisation muséale et en même temps pleinement exploitables, pour porter un discours promotionnel sur l'identité d'une entreprise.

Plus globalement, le principe de jeu d'association symbolique est aussi l'une des caractéristiques du discours publicitaire. Si les communications promotionnelle et muséale diffèrent entre elles 
par les techniques et codes qu'elles utilisent, leur fonctionnement sémiotique les rapproche et rend possible leur mélange et cohabitation. "C'est parce qu'elle ne recourt pas aux moyens et aux canaux de communication classiques que la patrimonialisation se distingue d'une communication publicitaire » (De La Broise, 1996, p. 93). Toutefois, actuellement, créer de l'intimité et de l'affection envers une marque, une entreprise ou ses produits est un enjeu stratégique pour les organisations marchandes qu'est à même de réaliser la patrimonialisation.

L'intérêt pour une entreprise repose en effet sur la possibilité de valoriser ses produits et marques tout en s'éloignant des codes traditionnels de la publicité dont se méfient et sont lassés les publics. Grâce à son musée, l'entreprise fait son apologie, valorise ses produits, son savoir-faire, son histoire, son identité, en somme fait sa publicité, mais sans en avoir l'air. La muséographie et scénographie permettent de valoriser l'organisation et ses produits en l'inscrivant dans le domaine culturel et en l'extrayant de la transaction et consommation du quotidien. Cette double stratégie à la fois d'effacement et de maximisation publicitaires correspond au phénomène plus global de dépublicitarisation et d'hyperpublicitarisation (Berthelot-Guiet et al., 2013).

Les musées d'entreprise sont des exemples de l'hybridation de plus en plus forte entre secteur marchand et non marchand. Musée de marque et marque muséale cohabitent dans le domaine patrimonial, montrant l'importance de la porosité des discours qui a pour effet de diluer les objectifs économiques et patrimoniaux au point qu'il devient de plus en plus délicat de les compartimenter.

\section{Références}

Barthes, R. (1964) Éléments de sémiologie. Communications, 4, 91-135

Berthelot-Guiet, K., Marti de Montety, C. \& Patrin-Leclère, V. (2013) Entre dépublicitarisation et hyperpublicitarisation, une théorie des métamorphoses publicitaires. Semen, PUFC, 36, 53-68.

Cousserand, I. (2009) Musées d'entreprise : un genre composite. Communication et organisation, 35, 192-213.

Cousserand, I. (2016) La mosaïque des musées d'entreprise, Bulletin de l'amcsti. Disponible à : https://amcsti.fr/fr/bulletin/mosaique-musees-dentreprise/. 
Broise (de la), P. (1996) Protection et valorisation du patrimoine industriel : signes de reconnaissance pour l'entreprise. Recherches en communication, 6 .

Broise (de la), P. (1996) L'entrée en patrimoine des entreprises publiques. La lettre de l'OCIM, 48, 3-9.

Davallon, J. Le Don du patrimoine : une approche communicationnelle de la patrimonialisation. Paris, Hermes Science-Lavoisier.

Desvallées, A. \& Mairesse, F. (dir.) (2011) Dictionnaire encyclopédique de muséologie. Paris, Armand Colin.

Rasse, P.(1995)Les musées d'entreprise : quelle médiation de l'histoire? Communication et organisation, doi : 10.4000/communicationorganisation.1768

Renaud, L. (2014) L'écriture publicitaire responsable : expression d'un paradoxe sociétal. Dans Lépine V., Martin-Juchat F. \& Millet-Fourrier C. (sous la dir. de) Acteurs de la communication des entreprises et organisations, Grenoble, PUG, 7996.

Tobelem, J.-M. (2005) Le nouvel âge des musées. Les institutions culturelles au défi de la gestion, Paris, Armand Colin.

Riegl, A. (1903) Le culte moderne des monuments. Sa nature, son origine, (traduction Jacques Boulet).

*Crédits photo : Laure Marchis-Mouren, Lise Renaud, Master Stratégie du Développement Culturel, parcours Médiation de la Culture et du Patrimoine

\section{(ब) $\Theta \Theta$}

«Attribution - Pas d'Utilisation Commerciale - Pas de Modification 4.0 International» (CC BY-NC-ND) 